\title{
The stability analysis of two-wheeled vehicle model
}

\author{
Juraj Gerlici ${ }^{1}$, Vladimir Sakhno ${ }^{2}$, Alla Yefymenko $^{2}$, Vladimir Verbitskii ${ }^{3}$, \\ Alexandr Kravchenko ${ }^{4}$, Kateryna Kravchenko, ${ }^{1, *}$ \\ ${ }^{1}$ University of Žilina, Department of Transport and Handling Machines, Univerzitná 1, 01026 Žilina, \\ Slovak Republic \\ ${ }^{2}$ National Transport University, Department of Automobiles, Suvorova 1, 01010, Kiev, Ukraine \\ ${ }^{3}$ Zaporizhzhia State Engineering Academy, Department of Automation System Maintenance, Soborny \\ Ave. 226, 69006, Zaporizhzhia, Ukraine \\ ${ }^{4}$ Zhytomyr State Technological University, Department of Automobiles and Motor Industry, \\ Chernyakhovsky 103, 10005, Zhytomyr, Ukraine
}

\begin{abstract}
This research investigates the stability of a two-wheeled vehicle model on the basis of numerical determination of full range of eigenvalues of a linear approximation matrix in the vicinity of the rectilinear driving mode. The received result was checked by numerical integration of the initial equations system of the disturbed motion of the model. The discrepancy of two research techniques is explained by the specialty of the considered mathematical model in which two pairs of complex conjugate eigenvalues close to each other are realized, that explains the emergence of standard derivations at calculating their numerical determination. The model is asymptotically stable in the range much wider than an operational interval (up to $100 \mathrm{~m} / \mathrm{s}$ ). In order to provide more intensive dampening of initial disturbances, it is possible to introduce additional resilient and damping elements between the trucks and the body in the design of the wheeled vehicle that will counteract the yaw mode of trucks.
\end{abstract}

Keywords: wheeled vehicle, truck, guiding wheeled module, mathematical model

\section{Introduction}

Monorail vehicles are used in many countries. That provides safety work of transport systems in big cities. The increase of the population in metropolises of Ukraine was a cause of new problems in public transport loading level. To solve these problems it is possible to implement monorail systems that will have several benefits, such as compactness, the cost of implementation and the safety of passengers' transit. Based on the fact that monorail vehicle is rapid-transit, the crucial task of the investigation is the stability of its running. The practical interest is aroused to the monorail vehicle with means of support «Alweg», discussed in Ryan R. Kennedy's works and online www.STC-IN.com \& www.skytraincorp.com (2004) [1, 2]. In Sakhno's and Yefymenko's recent researches

\footnotetext{
*Corresponding author: Kateryna.Kravchenko@fstroj.uniza.sk

Reviewers: Milan Sága, Milan Nad'
} 
$(2014,2015,2016)[3,4,5]$ the structural features of a monorail vehicle (hereinafter referred to as a wheeled vehicle) were investigated, the base unit of which is a truck that moves on four carrying pneumatic tires.

Traffic safety from a convergence or tipover, relative to the overpass, is provided by the guiding wheels. The mathematical model of the wheeled vehicle motion is considered in Korotenko's (1984) [6] and Yefymenko's (2015) [7] works where the authors analyse the rectilinear motion of the car and the movement along the transitional curves of the overpass, taking into account the influence of the forces and withdrawal moments arising in the contact spot of the bearing wheel with the road surface, as well as the elastic forces and moments generated by the guiding wheels.

The self-sustained vibrations in above mentioned works were not analysed; the task of the research was to establish the fact of stability or instability by the linear approximation (Korotenko, 1984) [6].

The key feature of the wheeled vehicle construction considered in this work is the fact that each guiding truck has one traction wheel (Fig. 1). This design solution is proved by weight reduction and simplification of the wheeled vehicle operation. However, it can lead to undesirable self-sustained vibrations. Various approaches to the analysis of rail and wheel vehicles self-sustained vibrations are presented in the works by Domin (1984) and Lobas (1990) [8,9]. Sakhno (2016) [10] used the numerical and analytical method and obtained an assessment of self-sustained vibrations amplitudes of a truck model of the wheeled vehicle in the straight lines of the overpass. Based on the methods of determining the stationary modes of the model motion, supplemented by an algorithm for constructing the bifurcation set in conjunction with the Poincare Index method, the work (2013) [11] analysed the number of fixed modes, and the stability boundaries were defined in the plane of the controlled parameters.

The purpose of the article. The purpose of this article is to analyse the stability of the two-wheeled vehicle model in the range of operating speed and the rational choice of its design values.

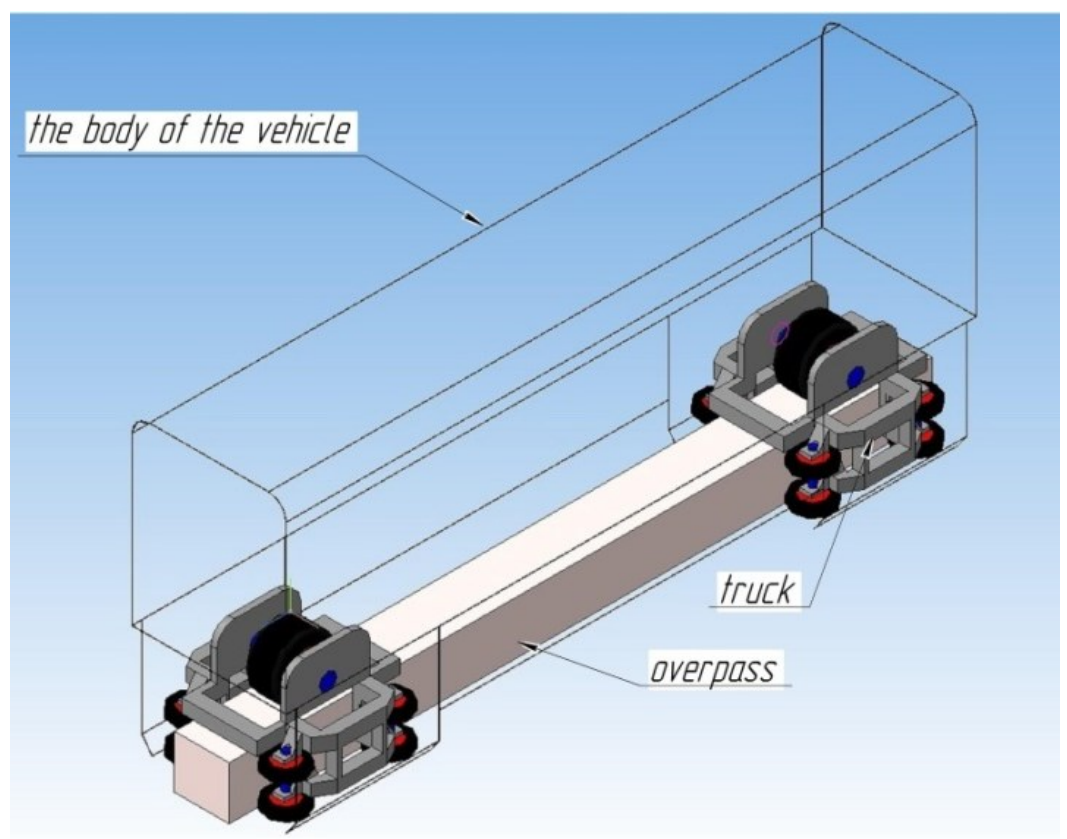

Fig. 1. Two-wheeled vehicle model 


\section{The stability analysis of straight-line motion of the two- wheeled vehicle model}

The wheeled vehicle consists of two trucks moving on pneumatical tires and performing the functions of traction wheels 1 and guiding wheels 2 (Fig. 2). The initial set of equations of the "two-wheeled" vehicle motion (1) on a straightway overpass is given relative to the inertial coordinate system moving with uniform velocity along the overpass centerline (Fig. 3).

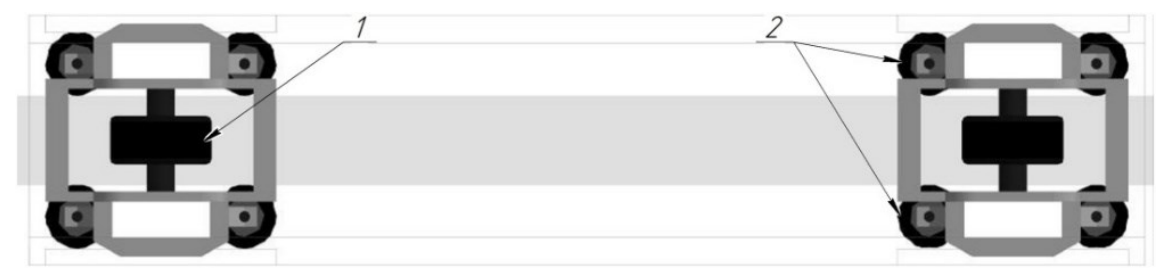

Fig. 2. The scheme of the wheeled vehicle: 1 - traction wheels, 2 - guiding wheels

\section{The body of the vehicle}

Overpass

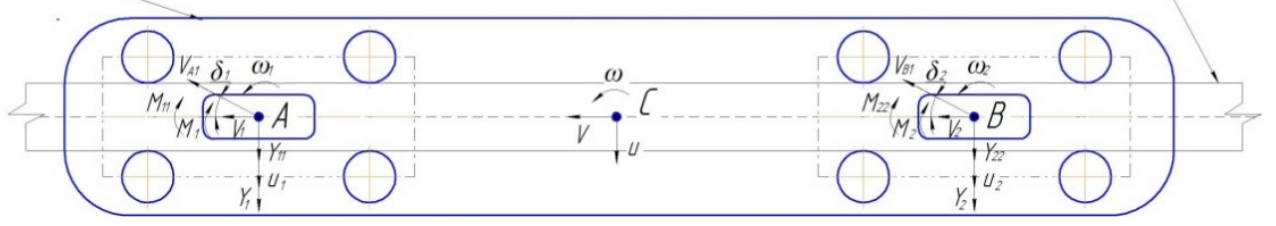

Fig. 3. Calculated bicycle scheme of the wheeled vehicle with two trucks

It is accepted in the work that the body position of the wheeled vehicle is calculated by the position of its mass center $\left(\mathrm{x}_{\mathrm{C}}, \mathrm{y}_{\mathrm{C}}\right)$ and by the heading angle $\psi$, the position of the trucks is calculated by the rotation angles $\psi_{1}$ and $\psi_{2}$, relative to the axis of the wheeled vehicle body.

The dynamic equations of the body plane-parallel motion are described by two half degrees of freedom: the transverse component of the mass center velocity $u$ and angular velocity relative to the vertical axis $\omega$.

The external forces and moments that have effect on the wheeled vehicle:

- the reduced elastic force $Y_{i}$ and the elastic moment $M_{i}$ caused by the guiding module from the overpass correspond to the ideas accepted before (Yefymenko, 2015 and Korotenko, 1984);

- forces $Y_{i i}$ and moments $M_{i i}$ of the traction wheel slip ( $i$ - truck number);

- $\quad X_{i i}$ - longitudinal forces in a spot of the traction wheels contact.

In rotational articulation (cylindrical joints) of the truck with the wheeled vehicle body the internal forces appear, which are formally withdrawn at the derivation of the equations of the whole system motion.

Thus, the equations of plane-parallel motion of the system, assuming that the longitudinal component of the vehicle mass center (parameter v) is kept constant, are as follows:

$$
\begin{aligned}
& -m_{1}\left(V_{1}-\omega_{1} u_{1}\right)-\mathrm{XA} \cdot \cos \psi_{1}+X_{11}-\mathrm{YA} \cdot \sin \psi_{1}=0 ; \\
& -m_{1}\left(U_{1}+\omega_{1} v_{1}\right)+\mathrm{XA} \cdot \sin \psi_{1}-\mathrm{YA} \cdot \cos \psi_{1}+Y_{11}+Y_{1}=0 ;
\end{aligned}
$$




$$
\begin{gathered}
-m_{2}\left(V_{2}-\omega_{2} u_{2}\right)-\mathrm{XB} \cdot \cos \psi_{2}+X_{22}-\mathrm{YB} \cdot \sin \psi_{2}=0 \\
-m_{2}\left(U_{2}+\omega_{2} v_{2}\right)+\mathrm{XB} \cdot \sin \psi_{2}-\mathrm{YB} \cdot \cos \psi_{2}+Y_{22}+Y_{2}=0 .
\end{gathered}
$$

The unknown reactions in rotational articulations with the wheeled vehicle body YA, $\mathrm{XA}, \mathrm{YB}, \mathrm{XB}$ are calculated by the set of equations (1) of the plane-parallel motion of the guiding trucks.

$$
\begin{gathered}
\mathrm{XA}=\frac{-\cos \psi_{1} \cdot m_{1} \omega_{1} \mathrm{u}_{1}-\sin \psi_{1} m_{1} \omega_{1} \mathrm{v}_{1}+\cos \psi_{1} \mathrm{~V}_{1} m_{1}-\sin \psi_{1} \mathrm{U}_{1} m_{1}-\cos \psi_{1} X_{11}+\sin \psi_{1} Y_{1}+\sin \psi_{1} Y_{11}}{\cos \psi_{1}^{2}+\sin \psi_{1}^{2}} ; \\
\mathrm{XB}=-\frac{-\cos \psi_{2} m_{2} \omega_{2} \mathrm{u}_{2}-\sin \psi_{2} m_{2} \omega_{2} \mathrm{v}_{2}+\cos \psi_{2} \mathrm{~V}_{2} m_{2}-\sin \psi_{2} \mathrm{U}_{2} m_{2}-\cos \psi_{2} X_{22}+\sin \psi_{2} Y_{2}+\sin \psi_{2} Y_{22}}{\cos \psi_{1}^{2}+\sin \psi_{1}^{2}} ; \\
\mathrm{YA}=\frac{-m_{1} \omega_{1} \mathrm{v}_{1} \cos \psi_{1}+\sin \psi_{1} m_{1} \omega_{1} \mathrm{u}_{1}+\mathrm{U}_{1} m_{1} \cos \psi_{1}+\sin \psi_{1} \mathrm{~V}_{1} m_{1}-Y_{1} \cos \psi_{1}-Y_{11} \cos \psi_{1}-\sin \psi_{1} X_{11}}{\cos \psi_{1}^{2}+\sin \psi_{1}^{2}} ; \\
\mathrm{YB}=-\frac{m_{2} \omega_{2} \mathrm{v}_{2} \cos \psi_{2}-\sin \psi_{2} m_{2} \omega_{2} \mathrm{u}_{2}+\mathrm{U}_{2} m_{2} \cos \psi_{2}+\sin \psi_{2} \mathrm{~V}_{2} m_{2}-Y_{2} \cdot \cos \psi_{2}-Y_{22} \cdot \cos \psi_{2}-\sin \psi_{2} \cdot X_{22}}{\cos \psi_{1}^{2}+\sin \psi_{1}^{2}} .
\end{gathered}
$$

where $\omega$ - the vehicle body angular velocity with according to the vertical axis, $\psi_{1}$ - relative angular velocity of the $i$-th truck, $m$ - mass of the vehicle body, $m_{i}$ - mass of the $i$-th truck.

The main kinematic relations used in the research are given below. The angular velocity of the guiding wheeled module is:

$$
\omega_{1}=\omega+\psi_{1}, \omega_{2}=\omega+\psi_{2}
$$

The angular acceleration of the guiding wheeled module is:

$$
\Omega_{1}=\Omega+\psi_{1}, \Omega_{2}=\Omega+\psi_{2}
$$

where $\Omega$ - the angular acceleration of the vehicle body.

The determination of lateral and longitudinal projections of the mass center velocity of the guiding wheeled module " $\mathrm{A}$ " was calculated using the following equations:

$$
\begin{gathered}
u_{1}=-v \cdot \sin \psi_{1}+(u+\mathrm{LA} \cdot \omega) \cos \psi_{1} \\
v_{1}=v \cdot \cos \psi_{1}+(u+\mathrm{LA} \cdot \omega) \sin \psi_{1} .
\end{gathered}
$$

The determination of lateral and longitudinal projections of the mass center velocity of the guiding wheeled module " $\mathrm{B}$ " was calculated by $(5)$ :

$$
\begin{gathered}
u_{2}=-v \cdot \sin \psi_{2}+(u-\mathrm{LB} \cdot \omega) \cos \psi_{2} ; \\
v_{2}=v \cdot \cos \psi_{2}+(u-\mathrm{LB} \cdot \omega) \sin \psi_{2} .
\end{gathered}
$$

LA, LB is the distance from the car mass center to the hinges "A" and "B".

The determination of lateral and longitudinal projections of the mass center acceleration of the guiding wheeled modules:

$$
\begin{gathered}
U_{1}=-V \cdot \sin \psi_{1}-v \cdot \cos \psi_{1} \cdot \psi_{1}+(U+\mathrm{LA} \cdot \Omega) \cdot \cos \psi_{1}+(u+\mathrm{LA} \cdot \omega) \cdot \sin \psi_{1} \cdot \psi_{1} \\
V_{1}=V \cdot \cos \psi_{1}-v \cdot \sin \psi_{1} \cdot \psi_{1}+(U+\mathrm{LA} \cdot \Omega) \cdot \sin \psi_{1}+(u+\mathrm{LA} \cdot \omega) \cdot \cos \psi_{1} \cdot \psi_{1}
\end{gathered}
$$




$$
\begin{gathered}
U_{2}=-V \cdot \sin \psi_{2}-v \cdot \cos \psi_{2} \cdot \psi_{2}+(U-\mathrm{LB} \cdot \Omega) \cdot \cos \psi_{2}+(u-\mathrm{LB} \cdot \omega) \cdot \sin \psi_{2} \cdot \psi_{2} \\
V_{2}=V \cdot \cos \psi_{2}-v \cdot \sin \psi_{2} \cdot \psi_{2}+(U-\mathrm{LB} \cdot \Omega) \cdot \sin \psi_{2}+(u-\mathrm{LB} \cdot \omega) \cdot \cos \psi_{2} \cdot \psi_{2}
\end{gathered}
$$

After the substitution of certain reactions (rotational articulations) in the equations of the plane-parallel wheeled vehicle motion, we have the closed equation system of motion $(8-9)$ :

$$
\begin{aligned}
& m(V-\omega u)=\mathrm{XA}+\mathrm{XB}=-\mathrm{V} m_{1}-\mathrm{V} m_{2}+\cos \psi_{2} X_{22}-\sin \psi_{2} Y_{2}-\sin \psi_{2} Y_{22}+\cos \psi_{1} X_{11}- \\
& -\sin \psi_{1} Y_{1}-\sin \psi_{1} Y_{11}+\mathrm{LA} \cdot m_{1} \omega^{2}-\mathrm{LB} \cdot m_{2} \omega^{2}+m_{1} \omega \mathrm{u}+m_{2} \omega \mathrm{u} \\
& m(\omega V+U)=\mathrm{YA}+\mathrm{YB}=Y_{1} \cos \psi_{1}+Y_{11} \cos \psi_{1}+\sin \psi_{1} X_{11}+Y_{2} \cos \psi_{2}+Y_{22} \cos \psi_{2}+ \\
& +\sin \psi_{2} X_{22}+\mathrm{LB} \cdot \Omega m_{2}-\mathrm{LA} \cdot \Omega m_{1}-m_{1} \omega \mathrm{v}-m_{2} \omega \mathrm{v}-\mathrm{U} m_{2}-\mathrm{U} m_{1} \\
& \mathrm{~J} \Omega= \\
& \quad-\mathrm{LA} \cdot m_{1} \omega \mathrm{v}+\mathrm{LB} \cdot m_{2} \omega \mathrm{v}+\mathrm{LB} \cdot \mathrm{U} m_{2}-\mathrm{LA} \cdot \mathrm{U} m_{1}-\mathrm{LB}^{2} \omega m_{2}-\mathrm{LA}^{2} \omega m_{1}- \\
& \quad-\mathrm{LB} \cdot \cos \psi_{2} Y_{2}-\mathrm{LB} \cdot \cos \psi_{2} Y_{22}-\mathrm{LB} \cdot \sin \psi_{2} X_{22}+\mathrm{LA} \cdot \cos \psi_{1} Y_{1}+ \\
& \quad+\mathrm{LA} \cdot \cos \psi_{1} Y_{11}+\mathrm{LA} \cdot \sin \psi_{1} X_{11}
\end{aligned}
$$

The equations of the truck guiding wheeled modules rotary motion:

$$
\begin{aligned}
& \mathrm{J}_{1} \Omega_{1}=M_{1}-M_{11} ;\left(\Omega_{1}=\Omega+\psi_{1}\right) ; \\
& \mathrm{J}_{2} \Omega_{2}=M_{2}-M_{22} ;\left(\Omega_{2}=\Omega+\psi_{2}\right) .
\end{aligned}
$$

$M_{1}$ - the total moment of elastic lateral reactions on the i-th truck;

$M_{11}$ - the moment of deviation of the carrier wheel on the i-th truck;

$J_{1}$ - the central moments of inertia of the vehicle body with respect to the vertical axis.

$J_{2}$ - the central moments of inertia of the truck with respect to the vertical axis.

Forces in a coupling point "A" (projections to longitudinal axes and lateral axes):

$$
\begin{gathered}
\mathrm{XA}=\mathrm{LA} \cdot m_{1} \cdot \omega^{2}+m_{1} \cdot \omega \cdot \mathrm{u}-\mathrm{V} \cdot m_{1}+\cos \psi_{1} \cdot X_{11}-\sin \psi_{1} \cdot Y_{1}-\sin \psi_{1} Y_{11} \\
\mathrm{YA}=-\mathrm{LA} \Omega m_{1}-m_{1} \omega \mathrm{v}-\mathrm{U} m_{1}+Y_{1} \cdot \cos \psi_{1}+Y_{11} \cos \psi_{1}+\sin \psi_{1} \cdot X_{11} .
\end{gathered}
$$

The equations of the dynamics of the plane-parallel motion of a wheeled vehicle assuming that the system maintains a constant value of the longitudinal component of the body mass center velocity (parameter v) and there are no longitudinal forces $X_{i i}$ (LA, LB is the distance from the car mass center to the hinges "A" and "B" ) are written in the form:

$$
\begin{gathered}
m(\omega v+U)=Y_{1} \cdot \cos \psi_{1}+Y_{11} \cdot \cos \psi_{1}+\sin \psi_{1} \cdot X_{11}+Y_{2} \cdot \cos \psi_{2}+Y_{22} \cdot \cos \psi_{2}+ \\
+\sin \psi_{2} \cdot X_{22}+\mathrm{LB} \cdot \Omega \cdot \mathrm{m}_{2}-\mathrm{LA} \cdot \Omega \cdot m_{1}-m_{1} \omega \mathrm{v}-m_{2} \omega \mathrm{v}-\mathrm{U}_{2}-\mathrm{U}_{1} \\
\mathrm{~J} \Omega=-\mathrm{LA} \cdot m_{1} \omega \mathrm{v}-\mathrm{LB}^{2} \cdot \Omega m_{2}-\mathrm{LA}^{2} \cdot \Omega m_{1}+\mathrm{LB} \cdot \mathrm{U} m_{2}+\mathrm{LB} \cdot m_{2} \omega \mathrm{v}-\mathrm{LB} \cdot \cos \psi_{2} \cdot Y_{2}- \\
-\mathrm{LB} \cdot \cos \psi_{2} \cdot Y_{22}-\mathrm{LB} \cdot \sin \psi_{2} \cdot X_{22}+\mathrm{LA} \cdot \cos \psi_{1} \cdot Y_{1}+\mathrm{LA} \cdot \cos \psi_{1} \cdot Y_{11}+\mathrm{LA} \cdot \sin \psi_{1} \cdot X_{11}- \\
-\mathrm{LA} \cdot \mathrm{U} m_{1} ; \\
J_{1}\left(\Omega+\psi_{1}\right)=-M_{1}-M_{11} ; J_{2}\left(\Omega+\psi_{2}\right)=-M_{2}-M_{22} .
\end{gathered}
$$


Approximation of the nonlinear dependences of forces and withdrawal moments can be presented in the form:

$$
\begin{aligned}
& Y_{i i}=k_{i} \cdot \delta_{i} / \sqrt{1+\left(k_{i} \delta_{i} /\left(\varphi_{i} N_{i}\right)\right)^{2}} ; \\
& M_{i i}=A_{i} \cdot \delta_{i} /\left(B_{i} \delta_{i}^{4}+C_{i} \delta_{i}^{2}+1\right) ;
\end{aligned}
$$

$k_{i}$ - slip drag coefficient;

$\delta_{i}$ - slip angle of the traction wheel of the truck $i$;

$\varphi$ - coefficient of friction of a wheel in the transverse direction;

$N_{i}$ - load on the traction wheel of the truck i;

$A_{i}, B_{i}, C_{i}$ - coefficients that are determined empirically.

The slip angles on the traction wheels of the truck $i$.

$$
\delta_{1}=-\operatorname{arctg} \frac{-v \sin \psi_{1}+(\mathrm{LA} \omega+u) \cos \psi_{1}}{v \cos \psi_{1}+(\mathrm{LA} \omega+u) \sin \psi_{1}} ; \delta_{2}=\operatorname{arctg} \frac{v \sin \psi_{2}-(-\mathrm{LB} \omega+u) \cos \psi_{2}}{v \cos \psi_{2}+(-\mathrm{LB} \omega+u) \sin \psi_{2}} .
$$

The elastic forces $Y_{i}(15)$ and the elastic moment $M_{i}$ (16) linearly depend on the transverse displacement of the truck mass center relative to the program trajectory and the difference of the truck heading angles and the program curve respectively.

$$
\begin{gathered}
Y_{1}=-C_{y} \cdot y_{A} ; Y_{2}=-C_{y} \cdot y_{B} ; \\
x_{A}=x_{C}+\mathrm{LA} \cdot \cos \psi ; y_{A}=y_{C}+\mathrm{LA} \cdot \sin \psi ; \\
x_{B}=x_{C}-\mathrm{LB} \cdot \cos \psi ; y_{B}=y_{C}-\mathrm{LB} \cdot \sin \psi ; \\
M_{1}=C y \cdot l l^{2}\left(\psi+\psi_{1}\right) ; M_{2}=C y \cdot l l^{2}\left(\psi+\psi_{2}\right) ;
\end{gathered}
$$

$C_{y}$ - lateral stiffness of pneumatic wheels;

$x_{A}, y_{A}, x_{B}, y_{B}$-coordinates of the wheeled vehicle truck mass center;

$l l$ - base regulating wheeled module;

$\psi_{1}, \psi_{2}$ - heading angles of the trucks.

\section{Research results}

The paper analyzes the stability of the rectilinear motion regime of a two-wheeled vehicle model based on the numerical determination of the complete set of eigenvalues of the system of linear approximation equations for the numerical values of the design parameters of the system listed below:

$m_{l}=m_{2}=1320 \mathrm{~kg} ; m=16000 \mathrm{~kg} ; J_{l}=J_{2}=4224 \mathrm{~kg} \cdot \mathrm{m}^{2} ; J=274400 \mathrm{~kg} \cdot \mathrm{m}^{2} ; \mathrm{LA}=\mathrm{LB}=7 \mathrm{~m}$; $k_{l}=k_{2}=360000 \mathrm{~N} ; C_{y}=250000 \mathrm{~N} / \mathrm{m} ; g=9,8 \mathrm{~m} / \mathrm{s}^{2} ; N_{l}=N_{2}=45715 ; l l=1,5 \mathrm{~m} ; \varphi=0,8$.

The speed parameter was discretely varied to determine the flutter (oscillatory instability) boundary when one control parameter was changed. In the general case, a pair of complex conjugate eigenvalues goes over into the right half-plane. However, the results of numerical integration of the initial equations of the disturbed motion indicated the damping of the initial perturbations in the velocity range, which is much broader than the boundary of the oscillatory instability thus found.

The analysis of this discrepancy is based on the numerical integration of the wheeled vehicle system at the speed of $12.3 \mathrm{~m} / \mathrm{s}$ and $22.3 \mathrm{~m} / \mathrm{s}$ with a fixed initial disturbance at the heading angle $\psi_{2}=0.001 \mathrm{rad}$ (second truck). 
The position of the eigenvalues of the system of linear approximation in the complex plane at a velocity of $12.3 \mathrm{~m} / \mathrm{s}$ and $22.3 \mathrm{~m} / \mathrm{s}$ is shown in Fig. 3. a), b). The first value of the velocity parameter belongs to the stability interval, the second supposedly should belong to the area of the oscillatory instability.

Both in the first and in the second case there are two pairs of complex-conjugate roots, which differ little from each other. As it turned out, this fact is a characteristic feature of this system and remains valid in a fairly wide range of speeds, since the subsystems corresponding to the heading angles have practically identical partial circular frequencies, the latter are generated by the elastic moments of the guiding wheeled modules, and a small discrepancy between the partial frequencies is entered by the stabilizing moments $M_{11}$ and $M_{22}$. Thus, the presence of sufficiently close two pairs of eigenvalues (located near the imaginary axis) can lead to the emergence of an error at their numerical determination.

This conclusion is fully confirmed by the results of numerical integration of the initial system, presented in Figures 5-7. Indeed, they illustrate the well-known phenomenon of synchronization of oscillations of two subsystems with close eigenfrequencies, which is observed in a wide range of the velocity parameter; on a time interval of the order of several tens of seconds, energy is "pumped" from one subsystem to another against the background of dissipative processes that in the final analysis provides asymptotic stability to undisturbed rectilinear motion.

To demonstrate close vibration frequencies with respect to the variables $\psi_{1}, \psi_{2}$, the fragments of the combined integral curves $\psi_{1}(t), \psi_{2}(t)$ in a small time interval are represented in Fig. 7, and the integral curve with the phase variable $\psi_{1}$ corresponds to a harmonic with a smaller value of the amplitude.

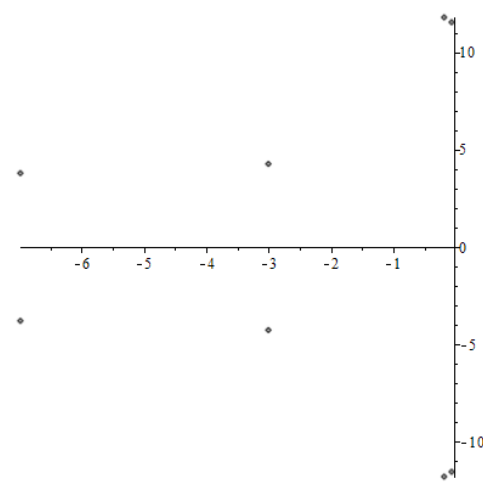

a)

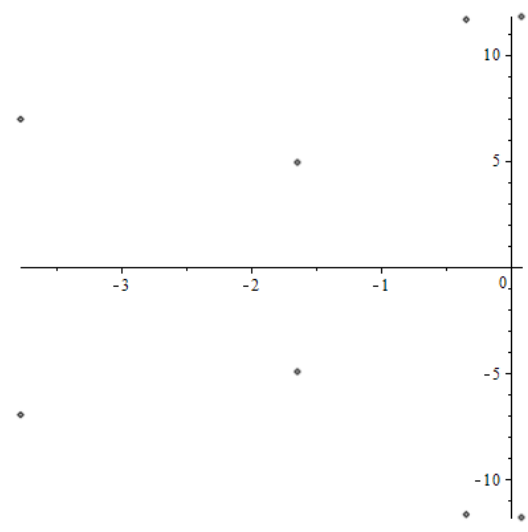

b)

Fig. 4. The position of eigenvalues of the linear approximation system near the rectilinear motion: a) $-v=12.3 \mathrm{~m} / \mathrm{s} ; \quad$ b) $-v=22.3 \mathrm{~m} / \mathrm{s}$ 


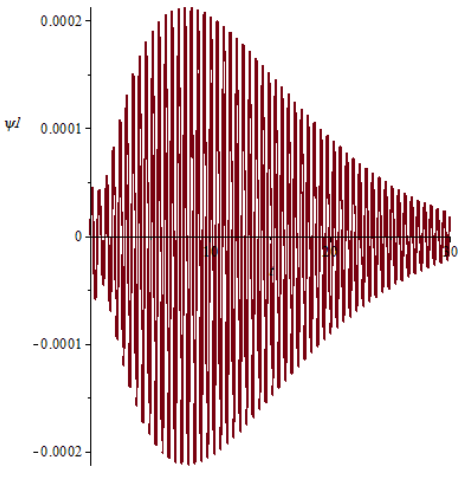

a)

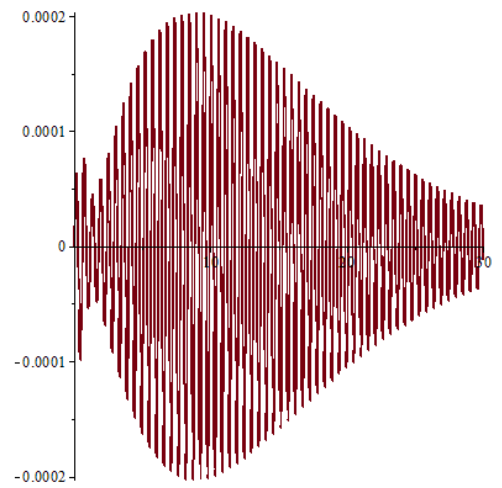

b)

Fig. 5. The heading angle of the first truck of the wheeled vehicle:

a) $-\mathrm{v}=12.3 \mathrm{~m} / \mathrm{s} ; \mathrm{b})-\mathrm{v}=22.3 \mathrm{~m} / \mathrm{s}$

At a speed of $v=22.3 \mathrm{~m} / \mathrm{s}$, based on the analysis of integral curves, a practical coincidence of the oscillation frequencies with respect to the variables $\psi_{1}, \psi_{2}$ is observed, which explains, in the final analysis, the cause of the error in the numerical determination of the set of eigenvalues.

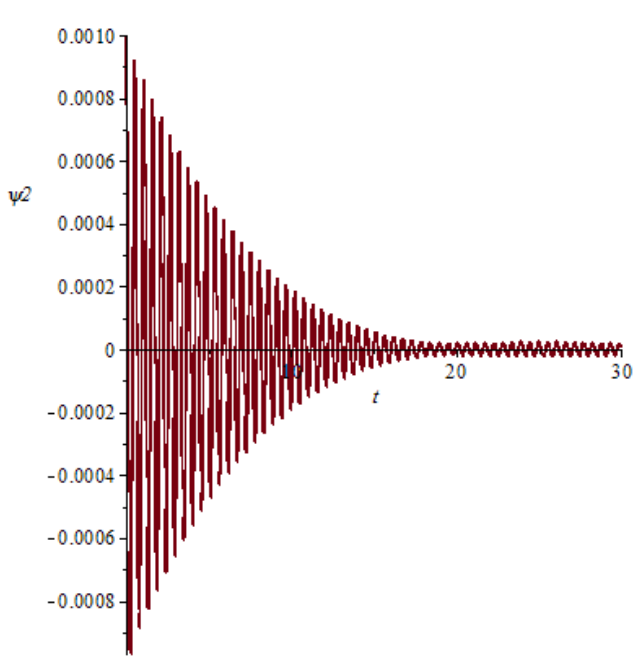

a)

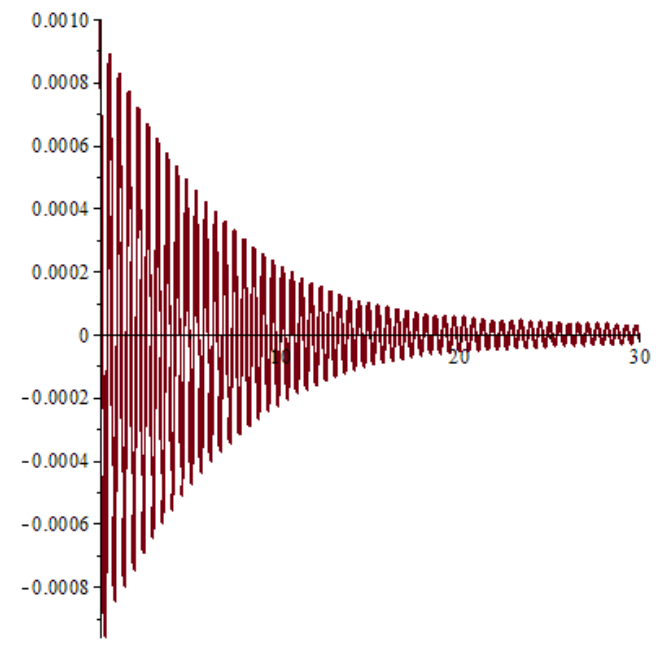

b)

Fig. 6. The heading angle of the second truck of the wheeled vehicle:

a) $-v=12.3 \mathrm{~m} / \mathrm{s} ; \mathrm{b})-v=22.3 \mathrm{~m} / \mathrm{s}$ 


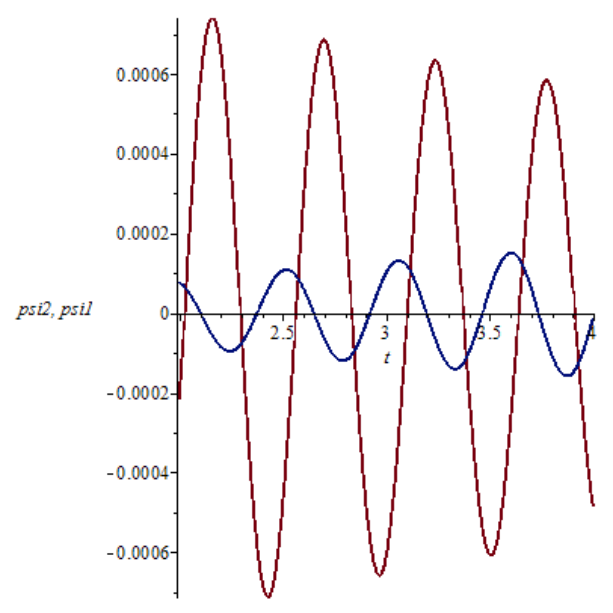

a)

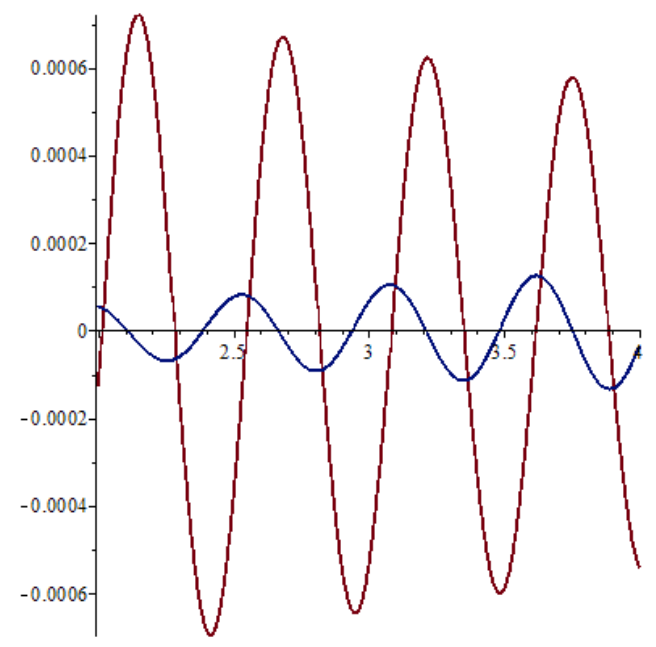

b)

Fig. 7. The heading angles of both trucks of the wheeled vehicle: a) $-v=12.3 \mathrm{~m} / \mathrm{s} ; \mathrm{b})-v=22.3 \mathrm{~m} / \mathrm{s}$

\section{Conclusions}

The presented plane (limited) model of a two-wheeled vehicle is asymptotically stable in a fairly wide range of speed (up to $100 \mathrm{~m} / \mathrm{s}$ ), however, as it can be seen from the results of numerical integration, the subsystems corresponding to the yawing angles of the trucks turned out to be synchronized. As a consequence, unwanted dynamic transient processes occur on the time intervals of the order of three to four tens of seconds, which can lead to some discomfort and fatigue of passengers. To ensure a more intensive attenuation of the initial disturbances, it is possible to introduce additional elastic and damping elements into the car's construction between the trucks and the body, which will parry the yawing of the trucks.

\section{References}

1. R. R. Kennedy, Considering Monorail Rapid Transit for North American Cities. available at: http://www.monorails.org/webpix\%202/RyanRKennedy.pdf (2004)

2. www.STC-IN.com \& www.skytraincorp.com, Monorail History and Technology of Successful Technology. available at: http://www.skytraincorp.com/pp/stc_mht.pdf (2004)

3. V. P. Sakhno, A. M. Yefymenko, About choosing the rational values of transverse stiffness parameters of the trolley guiding wheel module on pneumatic tires of the wheeled vehicle (analog of the system Alweg). The National Transport University Bulletin. Series: Technical Sciences, A Scientific and Technical Journal 2 (35), 204-210 (2016)

4. A. M. Yefymenko, A. V. Kostenko, Analysis of approximation methods of torque allocation hypotheses. SevNTU Bulletin: collection of scientific works, Series: Mechanical and instrument engineering 122/2011, 107-110 (2011)

5. A. M. Yefymenko, Analysis of the dynamics of a monorail van. Works of III International Scientific and Practical Internet Conference "Modern Technologies and 
Prospects of Motor Transport Development", April, 14-16, 2015: collection of scientific works / Vinnytsia National Technical University [and others], 62-66 (2015)

6. M. L. Korotenko, N.V. Dontsova Stability of the movement of a monorail elevated road van [Text]. Interuniversity collection of scientific works «Problems of the dynamics and strength of the railway rolling stock» DIIT 232, 53-58 (1984)

7. A. M. Yefymenko, V. H. Verbitsky, V. A. Demchenko, About the construction of mathematical model of a system Alweg monorail van. XXI Scientific and Technical Conference with International Participation "Transport, Ecology - Stable Development", (May 14-16, 2015, Varna), collection of reports, 341-346 (2015)

8. Y. V. Domin, L. A. Dluhach, M. L. Korotenko, O. M. Markova, Autooscillations of rail-tracked vehicles. 160 (1984)

9. L. H. Lobas, V. H. Verbitsky. Qualitative and analytical methods in the dynamics of wheeled machines. 232 (1990)

10. V. P. Sakhno, A. M. Yefymenko, About the oscillations analysis of a wheeled vehicle model at its moving along the straight overhead road. ZSTU Bulletin 2 (77), 248-256 (2016)

11. V. H. Verbitsky, V. P. Sakhno, A. P. Kravchenko, A. V. Kostenko, A. E. Danilenko Automobiles. Stability. Monograph, 176 (2013) 